\title{
SUBORDINATION FOR MEROMORPHIC HARMONIC FUNCTIONS
}

\section{J. DZIOK}

Abstract. We introduce and study classes of meromorphic harmonic functions defined by subordination. In addition to finding certain analytic criteria, we obtain some topological properties for the defined classes of functions. Some applications of these results are also given.

Mathematics subject classification (2010): 30C45, 30C80.

Keywords and phrases: Meromorphic harmonic functions, subordination, extreme points, starlike functions, convex functions.

\section{REFERENCES}

[1] O.P. Ahuja, J.M. JahangiRI, Certain meromorphic harmonic functions, Bull. Malays. Math. Sci. Soc. (2) 25 (2002), 1-10.

[2] I. Aldawish, M. DaRUs, On certain class of meromorphic harmonic concave functions, Tamkang J. Math. 46 (2015), 101-109.

[3] J. DZIoK, On Janowski harmonic functions, J. Appl. Anal. 21(2015), 99-107.

[4] W. Hengartner, G. Schober, Univalent Harmonic Functions, Trans. Amer. Math. Soc. 299 (1987), 1-31.

[5] J.M. JAhANGIRI, Harmonic meromorphic starlike functions, Bull. Korean Math. Soc. 37(2000), 291301.

[6] J.M. JahangiRI AND H. Silverman, Meromorphic univalent harmonic functions with negative coefficients, Bull. Korean Math. Soc. 36(1999), 763-770.

[7] W. JANOWS KI, Some extremal problems for certain families of analytic functions I, Annales Polonici Mathematici. 28(1973), 297-326.

[8] M. Krein, D. Milman, On the extreme points of regularly convex sets, Studia Mathematica 9(1940), $133-138$. 